\title{
Comparative Evaluation of Efficacy of Two New Commercially Available Desensitizing Agents: An in vivo Study
}

\author{
Sohani Maroli
}

\begin{abstract}
Objective: To compare the clinical efficacy of two commercially available desensitizing agents, Fluorprotector vivampuole and GLUMA comfort bond plus desensitizer in the treatment of dentinal hypersensitivity.
\end{abstract}

Materials and methods: A total of 203 teeth were selected for study. Patients who presented with a history of sensitivity to hot/cold, sweet, and sour food were examined using light tactile response along the cervical margin of the teeth. The assessment methods used to quantify sensitivity were tactile test, air blast test, and cold water test. The subjects were randomly divided into two groups. Group 1: GLUMA comfort bond plus desensitizer; group 2: Fluorprotector vivampuole (newly introduced by Ivoclar). The patients were evaluated for sensitivity at five appointments: at baseline, immediately after application of the agent, 1 day after application, after 1 week, and after 1 month.

Results: Patients belonging to group 2 showed significantly better results compared with patients of group 1 at 4 weeks.

Conclusion: Both the desensitizing agents showed significant reduction in sensitivity at all time intervals compared with baseline. However, Fluorprotector vivampuole appeared to be more effective in providing long-term relief against all the three test stimuli.

Clinical significance: Fluorprotector vivampuole is a newly introduced fluoride varnish which is clear and colorless. It can be used in cases of dentinal hypersensitivity without affecting the aesthetics of the patient, particularly in case of anterior teeth.

Keywords: Dentinal hypersensitivity, Desensitizing agents, GLUMA comfort bond plus desensitizer, Fluorprotector vivampuole.

How to cite this article: Maroli S. Comparative Evaluation of Efficacy of Two New Commercially Available Desensitizing Agents: An in vivo Study. Int J Experiment Dent Sci 2016;5(1):40-44.

Source of support: Nil

Conflict of interest: None

\section{Professor}

Department of Conservative Dentistry and Endodontics St. Joseph Dental College and Hospital, Eluru, Andhra Pradesh India

Corresponding Author: Sohani Maroli, Professor, Department of Conservative Dentistry and Endodontics, St. Joseph Dental College and Hospital, Eluru, Andhra Pradesh, India, Phone: 08212340441, e-mail: sohani_m@yahoo.com

\section{INTRODUCTION}

Dentin hypersensitivity $(\mathrm{DH})$ is a relatively common problem experienced in clinical dental practice. Dentin hypersensitivity is a painful clinical condition with an incidence ranging from $4-74 \%$. The variations in the reports may be because of difference in populations and different methods of investigations. Overall, it is estimated to affect about $15 \%$ of the general population to some degree. ${ }^{1}$

Several criteria are recognized as constituting an ideal desensitizing agent. They should be nonirritating to the pulp, painless and easy to apply, have rapid action, permanently effective, and maintain tooth esthetics. ${ }^{2}$ Numerous desensitizing agents ${ }^{1}$ are available with different modes of action like nerve desensitization, protein precipitation, or plugging of dentinal tubules. Glutaraldehyde, through its ability to precipitate salivary ${ }^{3}$ proteins in dentinal tubules, can be used to manage dentinal hypersensitivity. Use of fluoride as a topical desensitizing agent has been reported since 1941 by Lukomsky. ${ }^{4}$ Fluoride application leads to formation of calcium fluoride and, to some extent, also formation of fluoroapatite, thus mechanically blocking the transmission of stimulus to the pulp.

Fluorprotector vivampuole has been introduced in the market recently. It contains $0.1 \%$ fluoride and presents an easy-to-use snap-off feature. The present study was conducted to compare the in vivo efficacy of fluorprotector vivampuole with GLUMA comfort bond plus desensitizer.

\section{MATERIALS AND METHODS}

Patients recruited for the study were selected from the outpatient department of Conservative Dentistry and Endodontics. Patients reporting with a history of sensitivity to hot/cold, sweet/sour, mechanical stimuli on at least two teeth, having good general health, and no known allergy to test material were included in the study. Patients were in the age range between 20 and 65 years, with an average age of 52 years. Ethical clearance was obtained from the Institutional Ethical Clearance Board prior to the commencement of the study. 
Exclusion criteria were the presence of carious tooth/ teeth, restorations or cracked enamel, patients using desensitizing agent/dentifrices, those having a history of significant chronic systemic disease, and those under antibiotic or anti-inflammatory medication. ${ }^{5}$

After obtaining an informed written consent, the patients were examined by probing with light tactile pressure along the cervical margin of the teeth..$^{5} \mathrm{~A}$ total of 203 teeth were included in the study and the teeth were randomly divided into two groups: Group 1 comprised of 98 teeth, which were treated with GLUMA comfort bond plus desensitizer and group 2 comprised of 105 teeth, treated with Fluorprotector vivampuole.

\section{Clinical Examination}

Three test stimuli were applied after isolation using cotton rolls. The tests used were as follows: Tactile test: a mechanical stimulation was done along the cervical area carefully with the help of a sharp dental explorer. The explorer was passed lightly across the affected area, perpendicular to the long axis of the tooth. The test was repeated thrice before scoring by using discomfort scale ${ }^{6}$ and then the reading was noted.

Air blast test: A blast of air from the dental syringe was applied onto the affected area of the tooth isolated with cotton rolls, for 1 second from a standard distance of $10 \mathrm{~mm}$ (measured by taping scale to the dental syringe). The score was recorded using the discomfort scale.

Cold water test: Cold water was freshly melted within 1-2 minutes and it was then filled in precooled $1 \mathrm{ml}$ disposable syringe. After isolating the specific tooth, $0.2 \mathrm{ml}$ of this ice-cold water was slowly poured from the syringe on to the suspected tooth surface. ${ }^{6}$

The three test stimuli were applied in ascending order of discomfort, i.e., tactile test (least disturbing) then air blast test and at last cold water test (most disturbing). The stimuli were applied in the same order, at a time interval of 5 minutes each, at every recall visit. The patient response was recorded using the following scale: ${ }^{6}$

0 : No significant discomfort or awareness of stimulus;

1: Discomfort, but no severe pain;

2: Severe pain during application of stimulus;

3: Severe pain during and after application of stimulus.

Values of 2 and 3 were regarded as indicating hypersensitivity. ${ }^{7}$ Using the sensitivity measure, those teeth were selected which showed a score of $\geq 2$ for at least two test stimuli. The discomfort score was measured and recorded in a tabulated form to maintain a record. Improvement was defined as a change in score from 2 or 3 to 1 or 0 , indicating the improvement of the hypersensitive symptoms, and disappearance of symptom was indicated when the value changed from 2 or 3 to 0 .

\section{Group 1: GLUMA Comfort Bond + Desensitizer (Heraeus Kulzer, Hanau, Germany)}

The teeth were isolated with cotton rolls, cleaned and dried with cotton pellets. Hypersensitive tooth surface was conditioned with total etch technique using phosphoric acid etchant gel. Beginning with the enamel margins, etchant was applied to the entire cavity surface (including the dentin) and left for 20 seconds. Etchant was rinsed thoroughly and excess moisture was removed from the surface using a cotton pellet taking care not to dehydrate the dentin.

GLUMA comfort bond plus desensitizer was dispensed into a well. A soft disposable brush was soaked, and a copious amount was applied onto the entire dentin surface. Two additional coats of GLUMA comfort bond plus desensitizer were applied so that the whole dentin surface was uniformly shiny and left for 15 seconds. GLUMA comfort bond plus desensitizer was light-cured for 20 seconds with a standard light curing unit.

\section{Group 2: Fluorprotector VivAmpuole (Ivoclar, Vivadent, Ontario, Canada)}

The teeth were isolated with cotton rolls, cleaned, and dried with cotton pellets. Innovative "snap-off" feature of vivampoule enables the ampoule to be opened with ease. Specially provided Viva brushes (Ivoclar Vivadent, Ontario, Canada) were used to coat the varnish onto the affected tooth surface. The brushes were dipped into the ampuole and the surface of the tooth was thinly painted with varnish. The varnish was allowed to dry for 10-20 seconds, using a gentle blast from the air syringe. The patient was instructed not to rinse for at least 45 minutes after the application.

The teeth of both groups were evaluated the next day after treatment, at 1 week interval, and at 1 month interval using the three test stimuli.

\section{RESULTS}

The mean discomfort scores were compared between groups 1 and 2 at different time intervals using MannWhitney U-test. Tactile test showed no statistically significant difference between groups 1 and 2 at all the time intervals. Air blast test showed statistically significant difference at baseline, next day, and 1 week interval. Cold water test also showed statistically significant difference at 1 week and 1 month intervals. The mean discomfort scores after application of GLUMA comfort bond plus desensitizer and Fluorprotector vivampuole were compared individually at different time intervals using Kruskal-Wallis test. The results were highly significant for all the three test stimuli. 
Table 1 depicts the comparison of mean discomfort scores between groups 1 and 2 at different time intervals using Mann-Whitney U-test. Tactile test showed no statistically significant difference between groups 1 and 2 at all the time intervals. Air blast test showed statistically significant difference at baseline, next day and 1 week interval. Cold water test also showed statistically significant difference at 1 week and 1 month intervals.

Table 2 depicts the mean discomfort scores at different time intervals after application of GLUMA comfort bond plus desensitizer (group 1) using KruskalWallis test. The scores showed that there was a statistically significant difference found to tactile, air blast, and cold water test stimuli at all the time intervals. The mean discomfort score was found to decrease in 1-day time interval. But there was statistically significant increase in the mean discomfort score after 1 month. Graph 1 also gives the similar comparison of mean discomfort scores at different time intervals after application of GLUMA comfort bond plus desensitizer.

Table 3 depicts the mean discomfort scores compared at different intervals after application of Fluorprotector vivampuole (group 2) using Kruskal-Wallis test.

Table 1: Comparison of mean discomfort scores at different time intervals using Mann-Whitney U-test

\begin{tabular}{llllll}
\hline & & & & Std. \\
& Groups & $N$ & Mean & deviation & p-value \\
\hline Tactile & GLUMA bond & 98 & 1.02 & 0.497 & 0.263 \\
baseline & Fluorprotector & 105 & 0.93 & 0.624 & \\
Tactile & GLUMA BOND & 98 & 0.00 & 0.000 & \\
next day & Fluorprotector & 105 & 0.01 & 0.098 & 0.334 \\
Tactile & GLUMA bond & 98 & 0.00 & 0.000 & \\
next week & Fluorprotector & 105 & 0.01 & 0.098 & 0.334 \\
Tactile & GLUMA bond & 98 & 0.00 & 0.000 & \\
1 month & Fluorprotector & 105 & 0.00 & 0.000 & 1.000 \\
Air blast & GLUMA bond & 98 & 2.09 & 0.324 & \\
baseline & Fluorprotector & 105 & 1.91 & 0.667 & $0.018^{*}$ \\
Air blast & GLUMA bond & 98 & 0.30 & 0.459 & \\
next day & Fluorprotector & 105 & 0.45 & 0.537 & $0.042^{*}$ \\
Air blast & GLUMA bond & 98 & 0.32 & 0.467 & \\
next week & Fluorprotector & 105 & 0.57 & 0.648 & $0.005^{*}$ \\
Air blast & GLUMA bond & 98 & 0.37 & 0.485 & \\
1 month & Fluorprotector & 105 & 0.43 & 0.516 & 0.423 \\
Cold water & GLUMA bond & 98 & 2.40 & 0.492 & \\
baseline & Fluorprotector & 105 & 2.30 & 0.517 & 0.423 \\
Cold water & GLUMA bond & 98 & 0.62 & 0.584 & \\
next day & Fluorprotector & 105 & 0.83 & 0.672 & 0.182 \\
Cold water & GLUMA bond & 98 & 0.67 & 0.570 & \\
next week & Fluorprotector & 105 & 0.98 & 0.747 & $0.031^{*}$ \\
Cold water & GLUMA bond & 98 & 0.72 & 0.552 & \\
1 month & Fluorprotector & 105 & 0.70 & 0.606 & $0.003^{*}$ \\
\hline statically & sigifant & & & &
\end{tabular}

*Statistically significant
Table 2: Comparison of mean discomfort scores at different time intervals after application of GLUMA comfort bond plus desensitizer, with three different stimuli using Kruskal-Wallis test

\begin{tabular}{llllll}
\hline & & $N$ & Mean & Std. deviation & $p$-value \\
\hline Tactile & Baseline & 98 & 1.02 & 0.497 & $0.001^{*}$ \\
& Next day & 98 & 0.00 & 0.000 & \\
& Next week & 98 & 0.00 & 0.000 & \\
& One month & 98 & 0.00 & 0.000 & \\
& Total & 392 & 0.26 & 0.507 & \\
Air blast & Baseline & 98 & 2.09 & 0.324 & $0.001^{*}$ \\
& Next day & 98 & 0.30 & 0.459 & \\
& Next week & 98 & 0.32 & 0.467 & \\
& One month & 98 & 0.37 & 0.485 & \\
& Total & 392 & 0.77 & 0.882 & \\
Cold water & Baseline & 98 & 2.40 & 0.492 & $0.001^{*}$ \\
& Next day & 98 & 0.62 & 0.584 & \\
& Next week & 98 & 0.67 & 0.570 & \\
& One month & 98 & 0.72 & 0.552 & \\
& Total & 392 & 1.10 & 0.928 &
\end{tabular}

*Statistically significant

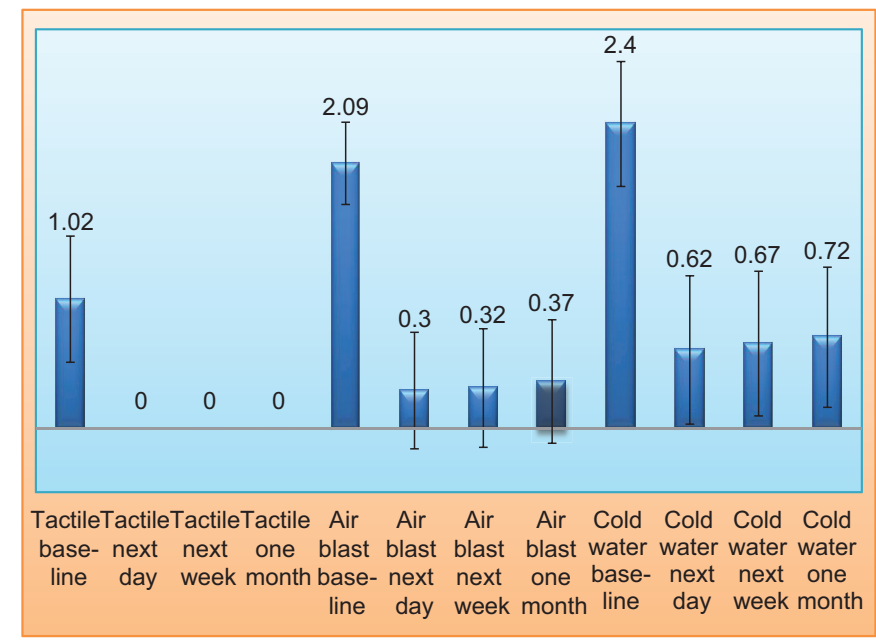

Graph 1: Comparing the mean discomfort scores after application of GLUMA comfort bond plus desensitizer at different time intervals

Statistically significant difference was found with tactile, air blast, and cold water test stimuli at all the time intervals. Graph 2 shows that a statistically significant decrease in mean discomfort scores was found the next day after the application of Fluorprotector vivampuole. One week after application, the scores increased. This is followed by a decrease in the scores 1 month after application, which were also statistically significant.

\section{DISCUSSION}

Dentinal hypersensitivity is described clinically as an exaggerated response to non-noxious sensory stimuli. It is a painful response of a tooth to different stimuli, such as dental brushing, food, or thermal changes. There are various diagnostic tools ${ }^{8}$ available for $\mathrm{DH}$ which include the air/water syringe (thermal), dental explorer (touch), percussion testing, bite stress tests, and other thermal 
Table 3: Comparison of mean discomfort scores at different time intervals with three different stimuli, after application of Fluorprotector vivampuole, using Kruskal-Wallis test

\begin{tabular}{llllll}
\hline & & $N$ & Mean & Std. deviation & $p$-value \\
\hline Tactile 2 & Baseline & 105 & 0.93 & 0.624 & $0.001^{*}$ \\
& Next day & 105 & 0.01 & 0.098 & \\
& Next week & 105 & 0.01 & 0.098 & \\
& One month & 105 & 0.00 & 0.000 & \\
& Total & 420 & 0.24 & 0.513 & \\
Air blast 2 & Baseline & 105 & 1.91 & 0.667 & $0.001^{*}$ \\
& Next day & 105 & 0.45 & 0.537 & \\
& Next week & 105 & 0.57 & 0.648 & \\
& One month & 105 & 0.43 & 0.516 & \\
& Total & 420 & 0.84 & 0.861 & \\
& Baseline & 105 & 2.30 & 0.517 & $0.001^{*}$ \\
& Next day & 105 & 0.83 & 0.672 & \\
& Next week & 105 & 0.98 & 0.747 & \\
& One month & 105 & 0.70 & 0.606 & \\
& Total & 420 & 1.20 & 0.905 & \\
\hline
\end{tabular}

*Statistically significant

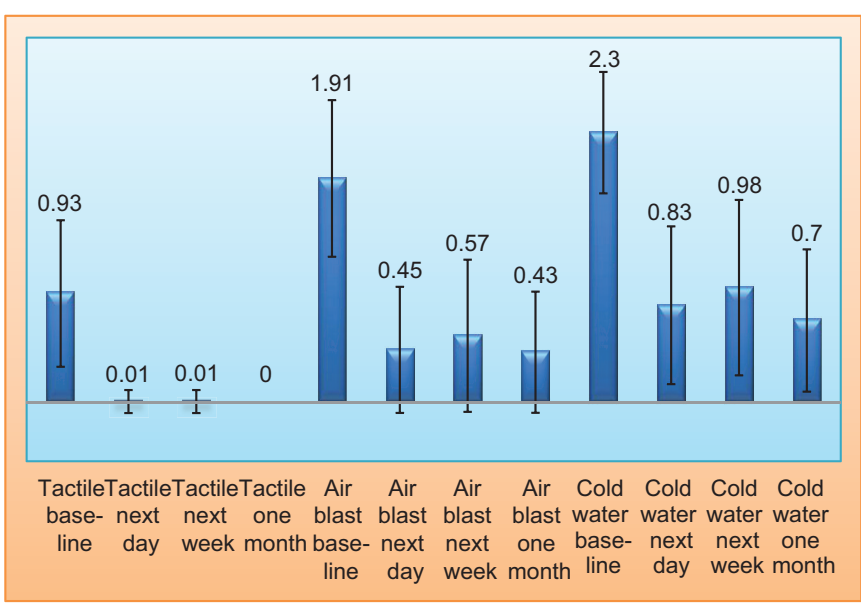

Graph 2: Comparing the mean discomfort scores after application of Fluorprotector vivampuole at different time intervals

tests such as an ice cube and assessment of occlusion. The three tests for DH used in this study are tactile test, air blast test, and cold water test. These tests were chosen because they are standard, easily performed, and sensitive. In the present study, it is obvious from the results that air blast and cold water tests were more effective at detection of hypersensitivity compared with tactile test.

GLUMA comfort bond plus desensitizer ${ }^{9}$ is an innovative combination of the single-component adhesive GLUMA comfort bond and GLUMA desensitizer. It is composed of methacrylate, 4-(2-methacryloxyethyl) trimellitic anhydride (4-META), ethanol, photoinitiators, and glutaraldehyde (2.5\%). In the reaction of glutaraldehyde ${ }^{3}$ with dentin, the two groups of aldehydes present in glutaraldehyde interlace themselves with the amino groups of dentin collagen, leading to a fixing of proteins, forming a barrier. The function of GLUMA ${ }^{10}$ as desensitizer to block dentinal tubules occurs via these two reactions:
1. Reaction of glutaraldehyde with part of serum albumin in dentinal fluid which induces a precipitation of serum albumin.

2. This reaction of glutaraldehyde with serum albumin is said to induce polymerization of 2-hydroxyethyl methacrylate.

Fluorprotector vivampuole ${ }^{11}$ is the new delivery form marketed by Ivoclar Vivadent. Each ampule contains $0.4 \mathrm{ml}$ and is composed of $1 \mathrm{gm}$ Fluor Protector which contains Bis\{4-[2-(difluorhydroxysilyl)ethyl]-2methoxycyclohexyl\} [N,N-(trimethylhexane-1,6-diyl) dicarbamate] (9 mg) (fluorsilane). This corresponds to $1 \mathrm{mg}$ fluoride. Auxiliary substances like ethylacetate, isopentyl propionate, and polyuria form the varnish. Varnish has $0.1 \%$ fluoride in a homogeneous solution which when dried has its concentration approximately $10 \times$ higher than the initial concentration. It has optimal flow and wetting properties allowing it to treat hard to reach areas. It is clear and colorless improving the esthetics and has excellent adhesion property.

Topical application of fluoride varnish causes precipitation of $\mathrm{CaF} 2$ onto exposed dentin surface which occlude the dentin tubules reducing the dentin permeability and further reducing $\mathrm{DH} .{ }^{5}$ The durability of the results in the present study is somewhat surprising because it might be expected that $\mathrm{CaF} 2$ precipitates formed on the outer dentin have been washed away by saliva and tooth brush abrasion, reopening the dentinal tubules and triggering hypersensitivity. Arends et $\mathrm{al}^{12}$ compared the efficacy of a fluoride varnish and Fluorprotector and confirmed that Fluorprotector has better penetration ability of up to $10 \mu \mathrm{m}$ depth compared with fluoride varnish which has less than $5 \mu \mathrm{m}$ penetration depth.

After application of either agent, both groups showed reduced discomfort scores on the first recall (1 day) with all the three different tests. At the second recall (1 week) with air blast and cold water tests, group 1 showed better results compared with group 2 . At the third recall (1 month), the difference between the two groups was not statistically significant, with air blast test. But with cold water test, group 2 showed better results compared with group 1 .

When mean discomfort scores were compared at different time intervals for each group, group I showed a highly significant decrease in discomfort scores compared with baseline values, the next day after application of GLUMA comfort bond plus desensitizer with all the three tests. It was observed that the discomfort scores increased significantly with time (1 week and 1 month) with both air blast and cold water tests.

Group 2 displayed a highly significant decrease in discomfort scores compared with baseline values, the next day after application of Fluorprotector vivampuole, 
with all the three tests. Slight increase in the discomfort scores was observed with air blast and cold water tests at the second recall (1 week). But decrease in the discomfort scores was observed at the third recall (1 month), with both air blast test and cold water test, proving a long-term relief from DH in group 2.

\section{CONCLUSION}

Both the agents showed significant reduction in sensitivity at different time intervals when compared with baseline values with all the three test stimuli. Both the agents used are potentially effective methods of managing the dentinal hypersensitivity. Among these two agents, Fluorprotector vivampuole appeared to be more effective in providing long-term relief compared with GLUMA comfort bond plus desensitizer.

\section{CLINICAL SIGNIFICANCE}

Fluorprotector vivampuole is a newly introduced fluoride varnish which is clear and colorless. It can be used in cases of dentinal hypersensitivity without affecting the esthetics of the patient, particularly in the case of anterior teeth.

Dentistry is varying with induction of modern science to practice dentistry. ${ }^{13}$

\section{REFERENCES}

1. Miglani S, Aggarwal V, Ahuja B. Dentin hypersensitivity: recent trends in management. J Conserv Dent 2010 OctDec;13(4):218-224.
2. Dababneh RH, Khouri AT, Addy M. Dentinehypersensitivityan enigma? A review of terminology, mechanisms, aetiology and management. Br Dent J 1999 Dec11;187(11):606-611.

3. Ishihata $H$, Shimauchi $H$, Kanehira M, Komatsu M, Finger WJ. Effects of glutaraldehyde, HEMA and gluma desensitizer on in vitro dentin permeability. Int J Contemp Dent 2011 Jan;2(1): 3-8.

4. Lukomsky EH. Fluorine therapy for exposed dentin and alveolar atrophy. J Dent Res 1941 Dec;20:649.

5. Ritter AV, Dias WL, Miguez P, Caplan DJ, Swift EJ Jr. Treating cervical dentin hypersensitivity with fluoride varnish—a randomized clinical study. J Am Dent Assoc 2006 Jul;137(7):1013-1020.

6. Pandit N, Gupta R, Bansal A. Comparative evaluation of two commercially available desensitizing agents for the treatment of dentinal hypersensitivity. Ind J Dent Res 2012 Nov-Dec;23(6):778-783.

7. Tarbet WJ, Silverman G, Stolman JM, Fratarcangelo PA. An evaluation of two methods for the quantitation of dentinal hypersensitivity. J Am Dent Assoc 1979 Jun;98(6):914-918.

8. Deepa KG, Sangeetha NU. Dentinal hypersensitivity and its management—an informed view. J Pharm Biomed Sci 2013 Feb;27(27):474-479.

9. Manufacturer's manual for gluma comfort bond plus desensitizer, Heraeus Kulzer. Available at: http://heraeuskulzer-us.com/media/webmedia_local/media/msds//

10. Bhandary S, Hegde MN. A clinical comparison of in-office management of dentin hypersensitivity in a short term treatment period. Int J Biomed Adv Res 2012 May;3(3):169-174.

11. Manufacturer's manual of fluorprotector vivampuole. Available at: http://www.ivoclarvivadent.us/en-us/products/ prevention-care/fluoridation/fluor-protector.

12. Arends J, Duschner H, Ruben JL. Penetration of varnishes into demineralised root dentine in vitro. Caries Res 1997;31(3): 201-205.

13. Saini R. Ozone therapy in dentistry: a strategic review. J Nat Sc Biol Med 2011;2:151-153. 